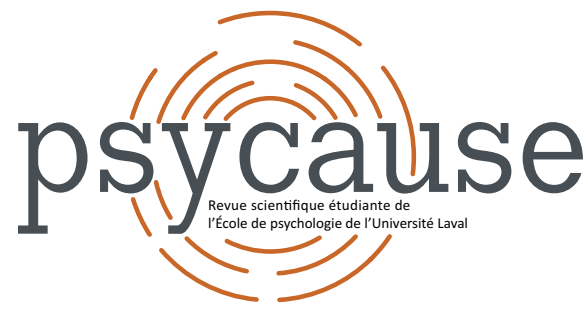

\title{
PSYCAUSE
}

Revue scientifique étudiante de l'École de psychologie de l'Université Laval

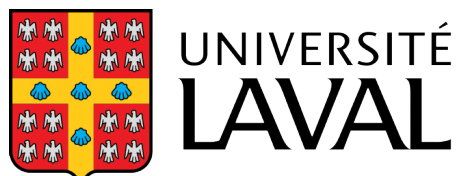

Faculté des sciences sociales École de psychologie

\section{BIAIS COGNITIFS FACE AUX CHANGEMENTS CLIMATIQUES}

Charlélie BÉNARD ${ }^{1, \forall, *}$, Agathe BLANCHETTE-SARRASIN ${ }^{1, \forall}$, Alessandro POZZ ${ }^{1, \forall}$, et François VACHON ${ }^{1}$

${ }^{1}$ École de psychologie, Université Laval

${ }^{\forall}$ Ces auteurs ont contribués également au manuscrit

*charlelie.benard.1@ulaval.ca

\section{Pour citer l'article}

Bénard, C., Blanchette-Sarrasin, A., Pozzi, A., \& Vachon, F. (2019). Biais cognitifs face aux changements climatiques. Psycause: Revue scientifique étudiante de l'École de psychologie de l'Université Laval, 9(2), 7-8. 


\title{
BIAIS COGNITIFS FACE AUX CHANGEMENTS CLIMATIQUES
}

\author{
Charlélie BÉNARD ${ }^{1, \forall, *}$, Agathe BLANCHETTE-SARRASIN ${ }^{1, \forall}$, Alessandro POZZI ${ }^{1, \forall}$, et François VACHON ${ }^{1}$ \\ 1 École de psychologie, Université Laval \\ ${ }^{*}$ Ces auteurs ont contribués également au manuscrit \\ *charlelie.benard.1@ulaval.ca
}

\section{Mots-clés : Biais cognitifs; Illusion d'empreinte écologique négative; Croyances compensatoires vertes; Changements climatiques.}

\begin{abstract}
Àl'heure de la lutte aux changements climatiques, les acteurs gouvernementaux tentent d'instaurer des politiques proenvironnementales afin de réduire l'empreinte écologique mondiale. Pourtant, plusieurs d'entre elles n'engendrent pas l'effet escompté, entraînant un sentiment d'urgence à comprendre les raisons pour lesquelles les populations n'y répondent pas adéquatement. Certains biais cognitifs permettraient d'expliquer l'inefficacité des politiques pro-environnementales: il apparaît donc nécessaire d'en approfondir la compréhension, puisque les mesures environnementales semblent insuffisantes à elles seules pour contrer les changements climatiques.
\end{abstract}

Kaklamanou, Jones, Webb et Walker (2015) suggèrent que l'origine des obstacles aux politiques pro-environnementales découle, du moins en partie, de l'existence de croyances compensatoires vertes (CCV). Ce biais cognitif se définit par la croyance que les conséquences positives d'un comportement écoresponsable (p. ex., recycler) compensent celles d'un comportement non écoresponsable (p. ex., ne pas faire de covoiturage). Ainsi, les personnes cherchent à disculper leurs actions non écoresponsables par les retombées positives de leurs comportements écoresponsables. Par exemple, une personne peut se déculpabiliser de prendre une douche plus longue qu'à l'habitude puisque l'eau utilisée provient de la récupération des eaux de pluie. De même, un autre biais cognitif peut venir entraver l'expérience pro-environnementale des individus. En effet, les gens sont disposés à croire qu'un élément vert est nécessairement bon pour l'environnement, alors qu'il est, en réalité, seulement moins nocif. Cette croyance amène les individus à endosser l'idée que l'ajout d'un élément vert permet de réduire l'empreinte écologique d'un ensemble d'éléments non verts (Holmgren, Kabanshi, Marsh \& Sörqvist, 2018). Or, en réalité, tout comportement, écoresponsable ou non, ajoute à l'empreinte environnementale. Cette évaluation fallacieuse, nommée l'illusion d'empreinte écologique négative (IEEN), amène les individus à consommer davantage que leurs besoins habituels.

Les CCV et I'IEEN pourraient expliquer partiellement l'inefficacité de certaines politiques environnementales. Toutefois,
I'origine de ces biais cognitifs est jusqu'à maintenant peu étudiée. Sörqvist et Langeborg (2019) ont récemment tenté d'établir un cadre théorique permettant d'expliquer leur origine, et proposent qu'ils découlent tous d'un mécanisme commun. Selon cette proposition, un biais de moyennage-où les répercussions environnementales d'un ensemble d'éléments ou d'actions sont estimées à partir de la moyenne des éléments, et non à partir de leur sommeserait à l'origine des deux biais. Bien qu'intéressante étant donné sa simplicité et sa parcimonie, cette proposition théorique n'a jamais été testée empiriquement. La présente étude vise à pallier cette lacune en ayant comme objectif de vérifier si les CCV et IEEN découlent d'un mécanisme commun. Si ces derniers découlent bel et bien du même biais de moyennage, leur magnitude respective devrait être corrélée positivement.

\section{Méthode}

Trois questionnaires sont administrés à 113 participant(e)s: deux évaluent les biais d'intérêt, soit l'IEEN et les CCV, et un mesure une variable contrôle, soit l'identité verte. Le questionnaire portant sur I'IEEN demande de faire l'évaluation de l'empreinte écologique de deux banlieues: une avec 150 bâtiments, et l'autre avec les mêmes 150 bâtiments additionnés de 50 maisons écoénergétiques (voir Holmgren et coll., 2018). Les participant(e)s doivent ensuite évaluer combien d'arbres il serait nécessaire de planter afin de compenser l'impact environnemental de la consommation énergétique de chacune des deux banlieues. La différence d'arbres obtenue entre l'évaluation des deux banlieues permet de déceler la magnitude du biais. En effet, si un individu indique que la banlieue comprenant des bâtiments écoénergétiques requiert moins d'arbres à planter pour compenser son impact environnemental que la banlieue sans bâtiments écoénergétiques, il manifeste alors I'IEEN. Les autres construits sont mesurés à l'aide de questionnaires comportant des énoncés sur une échelle Likert. Le questionnaire portant sur les CCV comporte 16 items (Kaklamanou et coll., 2015), et celui sur l'identité verte, mesurant les attitudes pro-environnementales d'un individu, en comporte quatre. 


\section{Résultats}

Contrairement à l'hypothèse selon laquelle il existe une corrélation positive entre les CCV et l'IEEN, aucune corrélation n'est observée entre les deux construits (voir Tableau 1). En effet, les données démontrent que, chez un même individu, un résultat élevé au questionnaire mesurant les CCV n'est pas nécessairement associé à la manifestation d'une forte IEEN. Ces résultats sont également appuyés par la comparaison des résultats aux $C C V$ entre les sujets qui présentent I'IEEN et ceux ne la présentant pas (voir Figure 1). Toutefois, la cote obtenue au questionnaire mesurant les CCV corrèle négativement avec celle obtenue au questionnaire portant sur l'identité verte: plus un individu se considère écoresponsable, moins celui-ci démontre de CCV.

\section{Tableau 1}

Matrice de corrélation de Pearson entre les résultats des trois questionnaires et statistiques descriptives de chacun des questionnaires

\begin{tabular}{lcccc}
\hline & IEEN & CCV & Moyenne & Écart-type \\
\hline IEEN & - & - & $-0,99$ & 18,72 \\
CCV & 0,03 & - & 30,18 & 8,86 \\
Identité Verte & 0,08 & $-0,20^{*}$ & 16,47 & 2,08 \\
\hline
\end{tabular}

${ }^{*} p<0,05$

\section{Discussion}

L'absence de relation entre les CCV et l'IEEN ne permet pas de fournir un appui empirique au modèle théorique de Sörqvist et Langeborg (2019) qui postule qu'un biais de moyennage est à l'origine des deux biais cognitifs. Les résultats proposent plutôt que les CCV et I'IEEN sont sous-tendues par des mécanismes différents. Alors que I'IEEN semble découler de l'application inappropriée d'une heuristique décisionnelle de moyennage (voir Holmgren et coll., 2018), les CCV trouveraient leur origine ailleurs. À cet égard, Kaklamanou et ses collègues (2015) proposent plusieurs hypothèses qui permettent d'expliquer la corrélation négative obtenue entre les CCV et l'identité verte. L'une d'elles avance qu'une forte identité verte réduit la propension à s'engager dans des activités non écoresponsables, diminuant ainsi les opportunités de présenter des CCV. Conséquemment, il se peut que l'identité verte annonce

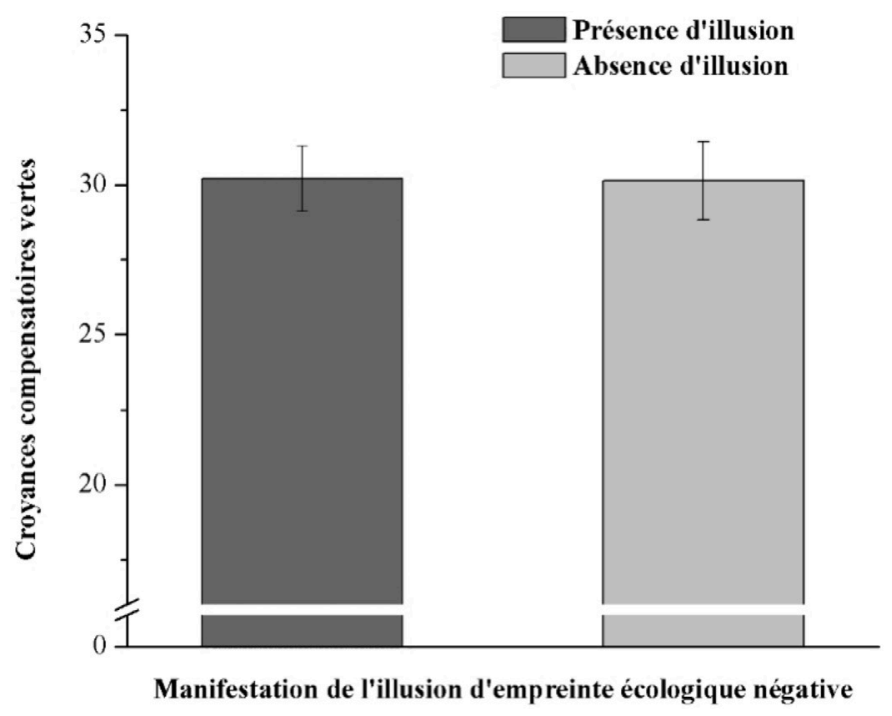

Figure 1. Comparaison de la manifestation au CCV en fonction de la présence de l'IEEN. Pour ce faire, les participant(e)s sont séparé(e)s en deux groupes, selon qu'ils ou elles manifestent $(N=52)$ ou non $(N=61)$ le biais de I'IEEN. Les données indiquent que le niveau de CCV ne diffère significativement pas entre les deux groupes, $t(111)=0,47, p=0,963$.

de manière plus adéquate la présence de CCV que ne le permet le biais de moyennage. Ainsi, il semble que la compréhension des biais cognitifs face aux changements climatiques apparaisse plus complexe qu'anticipé par Sörqvist et Langeborg.

\section{Références}

Holmgren, M., Kabanshi, A., Marsh, J. E., \& Sörqvist, P. (2018). When $A+B<A$ : Cognitive bias in experts' judgment of environmental impact. Frontiers in Psychology, 9, 823. doi:10.3389/fpsyg.2018.00823

Kaklamanou, D., Jones, C. R., Webb, T. L., \& Walker, S. R. (2015). Using public transport can make up for flying abroad on holiday: Compensatory green beliefs and environmentally significant behavior. Environment and Behavior, 47(2), 184204. doi:10.1177/0013916513488784

Sörqvist, P., \& Langeborg, L. (2019). Why people harm the environment although they try to treat it well: An evolutionary-cognitive perspective on climate compensation. Frontiers in Psychology, 10, 348. doi:10.3389/fpsyg.2019.00348

\section{Pour citer l'article}

Bénard, C., Blanchette-Sarrasin, A., Pozzi, A., \& Vachon, F. (2019). Biais cognitifs face aux changements climatiques. Psycause: Revue scientifique étudiante de l'École de psychologie de l'Université Laval, 9(2), 7-8. 